\title{
DYNAMIC EFFECTS IN THE MAIN LINAC OF CLIC
}

\author{
N. Leros, D. Schulte, CERN, Geneva, Switzerland
}

\begin{abstract}
The main linac of CLIC is very sensitive to jitters of the quadrupoles in transverse position and strength. Drifts due to the ground motion for example, have also to be considered, as well as errors on the amplitude and phase of the accelerating RF. This paper investigates the impact these dynamic effects have on the emittance and the luminosity of the collider, and the possibility to use feedbacks to correct the ground motion effect.
\end{abstract}

\section{INTRODUCTION}

Several dynamic effects in the main linac of CLIC [1], such as jitter effects or drifts, can reduce the luminosity. Jitter effects cannot be corrected and thus some estimation of the tolerances on the jitter amplitudes of different sources are discussed in section 2 . In contrast, drift effects can be corrected. The possibility to reduce the effect of ground motion with the help of feedbacks is considered in section 3. Finally, the luminosity loss due to ground motion is calculated taking into account beam-beam effects at the interaction point.

All results have been obtained using PLACET [2, 3], by simulating a single bunch beam containing $4 \times 10^{9}$ particles. The bunch length is $30 \mu \mathrm{m}$ and the vertical emittance is $\epsilon_{y}=5 \mathrm{~nm}$ at the entrance of the linac. The lattice of CLIC is described in [4] and [1].

Results concerning the vertical emittance are mainly presented since the effects are less critical in the horizontal plane.

\section{JITTER EFFECTS}

With jitter effects, the beam will vary from one pulse to the next. The relevant parameter for the luminosity is therefore the multi-pulse emittance, which is defined as the emittance of the ensemble of a large number of consecutive pulses.

A relative time jitter between the main beam and the drive beams leads to a jitter in the RF-phase, which changes the final beam energy and leads to a beam emittance growth. The effect has been simulated assuming that the change in phase is the same along the whole accelerator [5]. This could be due to a timing error of the main beam or more likely to a phase jitter of the drive beam at production. The power extraction takes place at a frequency which is 32 times higher than the production frequency of the drive beam. Therefore, a relative RF-phase error in the drive beams is multiplied by the same factor in the main beam. The maximum energy error acceptable at the end of the main linac is $\Delta E / E \leq 0.1 \%$, to stay in the energy acceptance of the final focus system [7]. Thus one has to keep the RF-phase jitter below $0.2^{\circ}$. The emittance growth due to such an RF-phase error is acceptable, with only $\Delta \epsilon_{y} / \epsilon_{y}=1 \%$. A coherent error of the gradient along the linac can be treated similarly. One must limit the gradient jitter to $\Delta G / G \leq 0.1 \%$, which results in an emittance growth below 5\%. Details of the simulation concerning all these effects can be found in [5].

Transverse position jitter of the quadrupoles also leads to a luminosity reduction. For an RMS jitter of $1 \mathrm{~nm}$, we find an increase of the multi-pulse emittances of $\Delta \epsilon_{x} / \epsilon_{x} \approx$ $0.05 \%$ and $\Delta \epsilon_{y} / \epsilon_{y} \approx 3.6 \%$.

Small variations of the quadrupole strengths $K$ can occur due to power supply ripples. These change the phase advance along the linac. Even in a machine that has been aligned using beam-based correction, the beam does not go through the centres of the quadrupoles. Consequently strength variations give rise also to transverse kicks. This effect has been simulated for the nominal CLIC alignment method described in [4]. First, all elements are pre-aligned using a system of wires and lasers [1]. Then, the beam position monitors (BPMs) and the quadrupoles are aligned with the ballistic method [6] (beam-based alignment). Finally, 10 emittance tuning bumps are used to further reduce the emittance growth.

Moving the quadrupoles mechanically during the ballistic alignment, rather than using dipole correctors, leads to the smallest sensitivity to the variation of the quadrupole field strength. Even for a large strength jitter of $\left\langle(\Delta K / K)^{2}\right\rangle^{1 / 2}=10^{-3}$, the observed growth of the multi-pulse emittances is only of $\Delta \epsilon_{x} / \epsilon_{x} \approx 0.6 \%$ and $\Delta \epsilon_{y} / \epsilon_{y} \approx 1.6 \%$. The relative emittance growth $\Delta \epsilon_{y} / \epsilon_{y}$ goes up to $2.8 \%$ if the quadrupoles can be moved only in steps of $0.5 \mu \mathrm{m}$.

\section{FEEDBACK CORRECTION}

The emittance growth induced by the ground motion can be reduced using trajectory feedbacks. These locally resteer the beam to the reference trajectory, i.e. the trajectory the beam had immediately after the initial beam-based alignment. In the present simulations, each feedback consists of 2 dipole correctors and 3 BPMs. The dipole correctors are located in two consecutive, vertically focusing quadrupoles. The BPMs are located in front of the second feedback quadrupole and in front of the next two vertically focusing quadrupoles. The beam offset with respect to the reference trajectory is determined by the BPMs and corrected by the dipole correctors. The feedbacks are sepa- 
rated by an equal number of quadrupoles along the linac.

Two different methods to simulate the performance of the feedbacks are considered in the following. Firstly, the perfect feedback model assumes that the re-steering of the beam is exact, because the BPMs have no resolution error, and can be done in a single pulse. It therefore predicts the optimum performance that can in principle be achieved with given feedbacks and dynamic errors.

In order to verify that the perfect feedback performance can be achieved in practice, a second more realistic model is also simulated. Here, the feedbacks are assumed to be independent and to not exchange information. The BPM resolution is taken to be $\sigma_{\text {res }}=100 \mathrm{~nm}$ [8]. Each feedback independently determines the necessary current in the dipole correctors in order to re-steer the beam back to the reference trajectory in the BPMs. Only a fraction (determined by the gain $g$ ) of the calculated correction is actually applied before the next pulse. This model is slightly pessimistic, since in practice a feedback may take advantage of the information from the feedbacks that are up-stream.

The independent feedbacks are expected to lead to a larger emittance growth than the perfect ones. They need some time to correct the ground motion effect, so that at any time the most recent contribution is only partially corrected. The finite BPM resolution leads to a slight missteering of the beam. Also the fact that each feedback does not take into account the response of the other feedbacks leads to more emittance growth. All these effects depend on the gain, and are visible after short times. For long times $t$, one expects the emittance growth $\Delta \epsilon_{y, i}$ to be

$$
\Delta \epsilon_{y, i}(t, g) \approx \Delta \epsilon_{y, p}(t)+\Delta \epsilon_{y, r}(g)
$$

where $\Delta \epsilon_{y, p}$ is the emittance growth with perfect feedbacks, and the additional contribution $\Delta \epsilon_{y, r}$ is due to the difference between the models.

The ground motion is modelled by moving the girders that support the beam line elements according to the ATL model [9] using $\mathrm{A}=0.5 \times 10^{-6}(\mu \mathrm{m})^{2} /(\mathrm{sm})$.

First, perfect feedbacks are considered. An initially perfectly aligned linac is moved according to 10 minutes of ground motion, then the feedbacks are simulated. Figure 1 shows the emittance growth, averaged over 100 machines, as a function of the number of feedbacks $N_{f}$. One finds $\Delta \epsilon_{y} / \epsilon_{y} \approx 130 \%$ for $N_{f}=10$ and $\Delta \epsilon_{y} / \epsilon_{y} \approx 10 \%$ for $N_{f}=40$. The emittance growth is roughly proportional to $1 / N_{f}^{2}$ for large $N_{f}$.

The case of 40 independent feedbacks is considered next. First, the optimum gain has to be determined. This is done by simulating the emittance growth for different gains, averaged over 50 machines, using the following procedure. Initially the linac is perfectly aligned. The feedbacks are switched on for 200 pulses (2s). Ground motion is applied between pulses. As can be seen in Fig. 2 (dots), the optimum gain is about 0.02 . In this case, the final emittance growth is $\Delta \epsilon_{y} \approx 1 \%$. At smaller gains the correction is too slow to be effective. For large $g$, a significant part of the emittance growth is due to the finite BPM resolution as

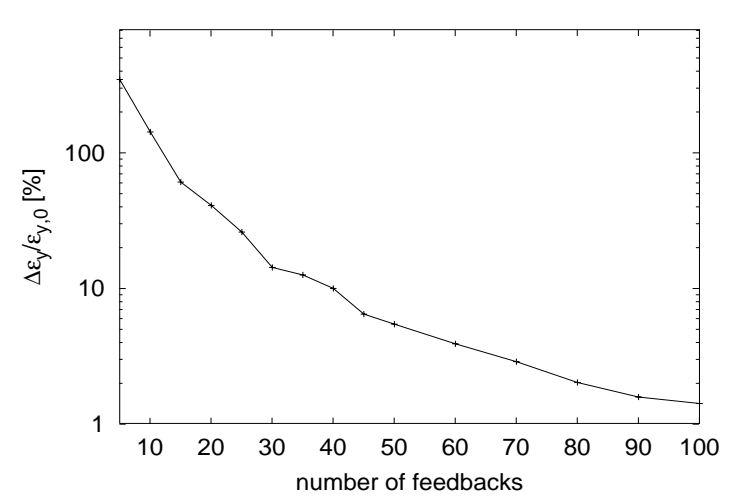

Figure 1: The relative emittance growth, after correction of a 10 minute ground motion, versus the number of feedbacks.

can be seen in Fig. 2 (open circles), which shows the case where no ground motion has been applied between pulses.

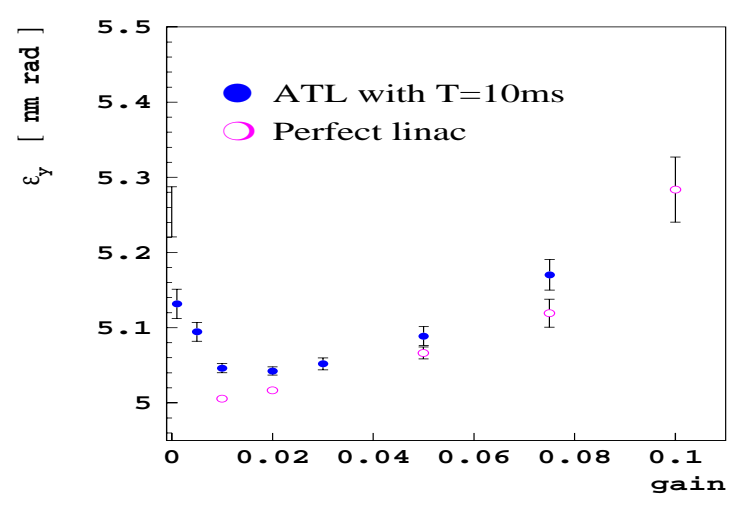

Figure 2: Emittance growth during $2 \mathrm{~s}$ versus the gain for a perfectly static linac (open circles) and in the presence of ground motion (dots).

The real-time simulation of the emittance growth shown in Fig. 3 helps to clarify the gain-dependent effects. Without feedbacks, the emittance grows by about $2.5 \%$ per second. With feedbacks used at the optimum gain $g=0.02$, the emittance first rises quickly, about as fast as with no feedbacks $(0.5 \mathrm{~s})$. On the time scale displayed, no growth is observed after this point. About half of the total emittance growth is due to the BPM resolution, as the case with no ground motion shows. With a small gain $g=0.001$, the emittance is still growing significantly after $2 \mathrm{~s}$. The feedbacks need a very long time to correct the ground motion, so the reduction of the growth rate is achieved very late. With a large gain $g=0.075$, the emittance rises initially even faster than without feedbacks. It reaches a small growth rate early, but at a high level. The optimum gain depends on the ground motion, a faster motion requiring larger gain.

After having shown the behaviour of the independent feedback model over very short times, we now consider 


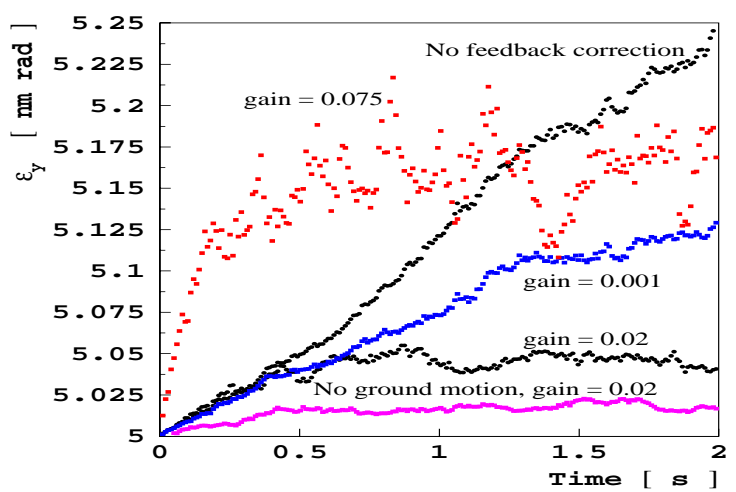

Figure 3: Time evolution of the emittance growth due to ground motion for different feedback gains and for a static linac (no ground motion) at the optimum gain of 0.02 .

the long time effects. Simulation of the $6 \times 10^{4}$ pulses that occur during 10 minutes is not possible. Thus the simulation is done using the following procedure. The linac is misaligned, corresponding to 10 minutes of ground motion. Then the feedbacks are switched on for 200 pulses. As before, ground motion is applied between pulses. Fig. 4 shows the emittance as a function of time. The final values are $\Delta \epsilon_{y} / \epsilon_{y}=8 \%$ for a gain $g=0.02$ and $\Delta \epsilon_{y} / \epsilon_{y}=$ $12 \%$ for a gain $g=0.1$. The results are consistent with the Eq. 1 within the statistical errors of the simulations, and as expected, $\Delta \epsilon_{y, r}(0.02)<\Delta \epsilon_{y, r}(0.1)$.

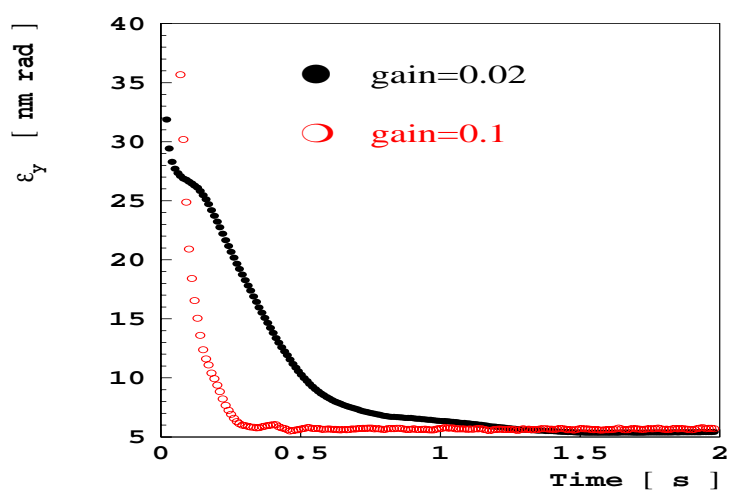

Figure 4: Time evolution of the emittance using 40 independent feedbacks and starting from a misaligned linac (10 minute ground motion).

\section{LUMINOSITY LOSS}

Up to this point, the emittance growth has been used as a measure for the luminosity loss. Now, the beam-beam effects will also be taken into account, but the effects of the beam delivery systems are neglected. First, we simulate the beam-based alignment for 100 different linacs, and find $\Delta \epsilon_{y} / \epsilon_{y} \approx 9 \%$. The final beams are taken pairwise as input to Guinea-Pig [10], a program that simulates the beam-beam interaction. With no beam-beam forces, the luminosity loss $\Delta L / L$ after beam-based alignment is $5.8 \%$ compared to the case with perfect linacs. If the beam-beam forces are taken into account, one finds $\Delta L / L=17.5 \%$.

In the next step, each corrected linac is moved according to 10 minutes of ground motion, and $N_{f}=30$ perfect feedbacks are applied; this results in $\Delta \epsilon_{y} / \epsilon_{y} \approx 24 \%$. With no beam-beam force the luminosity loss is $\Delta L / L=10.5 \%$, but with beam-beam force it is found to be $\Delta L / L=21 \%$. The additional luminosity loss due to the ground motion is $4.7 \%$ with no beam-beam forces and $3.5 \%$ with beambeam forces. While the loss due to static effects is strongly enhanced by the beam-beam interaction, the contribution from ground motion is slightly reduced. This underlines the fact that the emittance is not a very precise measure of the luminosity loss, and that finally a full simulation of linac, beam delivery system and beam-beam interaction will be needed.

\section{CONCLUSIONS}

The effects of different jitter sources have been discussed. One must limit a coherent jitter of the RF-phase and amplitude to $\Delta \phi \leq 0.2^{\circ}$ and $\Delta G / G \leq 0.1 \%$, respectively, to avoid large errors of the final beam energy. In this case, the emittance growth will also be acceptable. For the transverse positions and strengths of the quadrupoles, jitters of the order of $\Delta y \leq 1 \mathrm{~nm}$ and $\Delta K / K \leq 0.1 \%$ are acceptable.

A correction of the ground motion using feedbacks has been simulated. A simple optimistic model, that neglects dynamic effects during the correction, predicts that with 40 feedbacks the emittance growth can be limited to $10 \%$, after 10 minutes of ground motion. Also a more realistic model of independent feedbacks has been implemented in PLACET and simulated. With the optimum gain, the performance of the feedbacks in this model is close to the one with the perfect model. The influence of other error sources, e.g. beam jitter at injection, and of feedbacks in the beam delivery system, remains to be investigated.

\section{REFERENCES}

[1] CLIC Study Team, G. Guignard editor, CERN 2000-008 (2000).

[2] E.T. d'Amico, G. Guignard, N. Leros, D. Schulte, This conference.

[3] D. Schulte, Draft at http://home.cern.ch/dschulte/placet.html.

[4] D. Schulte, EPAC98, Stockholm, Sweden (1998).

[5] D. Schulte, CLIC-Note 432 (2000).

[6] T. Raubenheimer and D. Schulte, Proc. PAC99, New York, USA (1999).

[7] F. Zimmermann et al, CERN-SL-2000-057 (2000).

[8] J.P.H. Sladen, I. Wilson and W. Wuensch, CERN/PS-96-029 (LP).

[9] R. Assmann, C. Salsberg and C. Montag, Linac 96, Geneva, Switzerland (1996).

[10] D. Schulte, ICAP 1998 and CERN-PS-99-014 (LP) (1999). 